\title{
Coaching mit neuer transaktionsanalytischer Theorie
}

\author{
Günther Mohr ${ }^{1}$ \\ Angenommen: 20. Dezember 2021 / Online publiziert: 28. Januar 2022 \\ (c) Der/die Autor(en) 2022
}

\section{Zusammenfassung}

Transaktionsanalyse hat sich in ihren 70 Jahren Praxis und Theorieentwicklung zu einer sehr erprobten, integrativen, offenen Beratungstheorie entwickelt. Der Artikel zeigt wesentliche Aspekte transaktionsanalytischer Haltung und transaktionsanalytischer Vorgehensweise auf. Insbesondere ist die beziehungsorientierte Metatheorie dabei von großer Bedeutung. Auf die Strukturierung der Einzeltheorien der Transaktionsanalyse zu Menschenbild, Persönlichkeit, Beziehung und Kommunikation, Entwicklung und Veränderung, Wirklichkeit und Kontextbezug sowie Professionsmethoden zeigt ein durchaus komplexes Praxisbeispiel die Vielfalt des Interventionsspektrums auf.

Schlüsselwörter Beratung · Beziehung $\cdot$ Shadow-Coaching · Diamant der Professionalität · Organisationscoaching

\section{Coaching with new Transactional Analysis Theory}

\begin{abstract}
Transactional analysis has developed in its 70 years of practice and theory development into a highly tested, integrative, open theory of counseling. The article shows essential aspects of transactional analytic attitude and approach. In particular, the relational meta-theory is of great importance. On the structuring of the individual theories of transactional analysis on the image of man, personality, relationship and communication, development and change, reality and contextual reference as well as professional methods, a quite complex practical example shows the variety of the intervention spectrum.
\end{abstract}

Keywords Consulting $\cdot$ Relationship $\cdot$ Shadow coaching $\cdot$ Diamant of professionality $\cdot$ Organisational development

Der folgende Beitrag gibt einen Einblick in Theorie und Praxis des transaktionsanalytisch basierten Coachings. Die Methoden der Transaktionsanalyse (TA) wurden von Coaches sehr früh schon adaptiert, weil sie schnelle Veränderungen versprachen. In fünf Schritten werden im Folgenden Aspekte der Transaktionsanalyse für Coaching beschrieben. Den Anfang bilden einige grundsätzliche Bemerkungen zur aktuellen Transaktionsanalyse und ihrer Begegnung mit Coaching. Darauf folgt ein kurzer Überblick zum Forschungsstand, gefolgt von der beziehungsorientierten Metatheorie sowie von Einzelkonzepten im beraterischen Feld. Den Abschluss bildet eine Falldarstellung, ein MultilevelCoaching, das die vielfältigen Möglichkeiten transaktions-

Günther Mohr

hedmohr@aol.com

1 Klarastr. 7, 65719 Hofheim, Deutschland analytischer Methodik für die Praxis auch in komplexen Situationen andeutet.

\section{Transaktionsanalyse begegnet Coaching}

Die transaktionsanalytische Theoriebildung hat in den letzten Jahren sehr fruchtbare Ergebnisse gebracht. Dies hat auch mit Erfahrungen zu tun, die man in der praktischen Anwendung von Transaktionsanalyse gemacht hat. Der von Anfang an mehrperspektivische transaktionsanalytische Ansatz besaß mit Verhaltensorientierung, biografischtiefenpsychologischen und humanistisch-psychologischen Bestandteilen schon sehr gute Grundlagen für ein integrative Beratungsvorgehen. Zudem hat sich Transaktionsanalyse in nunmehr siebzig Jahren durch Praxis, Forschung und Theorieentwicklung stetig weiterentwickelt. Wie auch die Anwender anderer Kurzzeitansätze wie systemische Be- 
ratung oder NLP (Neuro-linguistisches Programmieren) mussten auch Transaktionsanalytiker erfahren, wie wichtig eine längere beraterische Begleitung sein kann und wie konzeptionell weitergespannt der Horizont gerade für Coaching im Organisationskontext sein muss.

Dadurch wurde die grundlegende und umfassende Beziehungsorientierung, die im Begriff Transaktion enthalten ist, noch deutlicher in den Vordergrund gerückt. Hinzu kam der Blick auf Systeme. Die Klienten in vielen Coachings sind Teil eines größeren Organisations- oder Unternehmenssystems. Dort ist die Ressourcenorientierung, also die Suche nach und die Entwicklung von Kompetenzen bedeutsam. Zudem ist das ethische Vorgehen in seinem besonderen Schwerpunkt der Transaktionsanalyse sicher noch einmal verstärkt in den Vordergrund gerückt, wie beispielsweise der neu eingeführte Ethik-Code zeigt (EATA-Ethical $\left.\mathrm{Code}^{1}\right)$. Aus der ehemals populären Kurzzeit-Psychotherapiemethode ist durch Forschung und Theoriebildung ein professioneller Ansatz geworden, der in vielen Ländern der Welt in den Feldern Therapie, Beratung, Coaching, Bildung und Organisationsentwicklung erfolgreich eingesetzt wird und dafür auch ein weltweit verbundenes, weitgehend einheitliches Qualifizierungssystem über die Verbände der internationalen TA-Gesellschaft ITAA (www.itaaorg. com) und der europäischen Dachgesellschaft EATA (www. eatanews.com) anbietet. Auf der Basis des zentralen Begriffs Beziehung gibt es einige Grundparameter für die Identität des Transaktionsanalytikers (Schmid 1989; Mohr 2005). Was macht die Praxis der Transaktionsanalyse aus und unterscheidet sie von anderen?

1. Professionelle Arbeit in Therapie, Beratung und Coaching, Organisationsentwicklung und Bildung basiert auf Transaktionen. „Transaktionsanalytiker gestalten Wirklichkeit durch Kommunikation" (Schmid 2003). Die Transaktion als Basiseinheit von Beziehung und Kommunikation stellt diagnostische Information zur Verfügung und das Medium zur Intervention dar.

2. Transaktionsanalyse ist ein humanistischer Ansatz, was bedeutet, dass man eine grundsätzlich wertschätzende, resourcenorientierte Haltung dem Klienten gegenüber einnimmt.

3. Transaktionsanalytiker nutzen und konstruieren zu Veranschaulichung und didaktischem Nutzung praktische Mustermodelle für soziale und gesellschaftliche Situationen.

4. Transaktionsanalytiker arbeiten experimentell. Sie beanspruchen nicht die Wahrheit, sondern arbeiten mit Hypothesen, die sie überprüfen und anpassen.

\footnotetext{
${ }_{1}^{1}$ EATA-Ethical code. https://eatanews.org/wp-content/uploads/2019/
} 04/ethics-code-feb-13th-edit.pdf. Zugegriffen: 25. Nov. 2021.
5. Transaktionsanalytiker beziehen sich auf einen Kontext. Beziehungen offenbaren je nach Kontext ein bestimmtes Rollengefüge und dadurch eine bestimmte Qualität. Rollenbeziehungen in der Privatwelt sind beispielsweise deutlich von Beziehungen in der Berufswelt zu unterscheiden.

6. Transaktionsanalytiker stehen über die International Transactional Analysis Association (ITAA) und die European Association for Transactional Analysis (EATA) in einem stetigen Diskurs zur Weiterentwicklung der Qualifizierungen für Therapie, Beratung, Coaching, Bildung und Organisationsentwicklung.

Mit diesen Charakteristika für eine Identität des Transaktionsanalytikers steht nicht die Nutzung der oft benannten Standardmodelle Ichzustände, Skript und Spiele im Vordergrund, sondern die Metaposition, die diese Modelle und viele andere auf der Basis einer humanistischen Beziehungshaltung nutzt.

Auf welche Coachingwelt trifft Transaktionsanalyse. Seit Wolfgang Looss' Ausrufung der Coachingwelt in Deutschland mit „Unter vier Augen“ im Jahre 1991 ist schon einige Zeit vergangen (Looss 1996). Es zeigen sich über die verschiedenen Phasen der Coachinganwendung hinweg mehrere Stränge der theoretischen Herleitung des Coachings. Da ist einmal die psychotherapeutische Tradition. Viele Coachingansätze und -methodiken haben ihren Ursprung in der Psychotherapie und auch viele der heute sehr etablierten Coaches haben noch rein psychotherapeutische Ausbildungen genossen. Dies reicht über alle großen Schulen hinweg. Transaktionsanalyse ist auch ein Beispiel davon. Die Coachingbücher waren auf dem Hintergrund der psychotherapeutischen Tradition lange Zeit so gegliedert, dass es zunächst mit Beiträgen aus bestimmten psychotherapeutischen Schulen begann. So wurden im Sammelband „Diagnostik im Coaching“ zu Beginn noch vier Schulen thematisiert: Psychodynamik, Psychodrama, Gestalt und kognitive Verhaltenstherapie (Möller und Kotte 2013). Auch der Coaching-Grundlagenband von Lippmann (2013) hat noch schulenspezifisches Herangehen in der Betrachtung. Dennoch geht der Weg in eine integrative Richtung. Die Beratungswissenschaften und auch Coaching sind auf dem Weg zu einer integrierten Form der Beratung (Roth und Ryba 2016). Dies bedeutet, dass sowohl aktuelle verhaltensbezogene Fokusse wie auch biografischtiefenpsychologische, systembezogene, zunehmend auch hypnosystemische Elemente eine integrierte Praxis von Coaches bestimmen (Ryba 2018).

Es gibt im Coaching allerdings auch die Tradition aus dem Kaufmännischen, der Betriebswirtschaft, der klassischen Unternehmensberatung, der Soziologie, der Philosophie und aus anderen Feldern (z. B. Rautenberg 2020). Dabei bleibt allerdings zu beachten, dass das Grundlegende 
am Entwicklen von Menschen zwischen Therapie und Coaching nach heutigem Stand nicht fundamental unterschiedlich ist, sondern im Grundsätzlichen eher in ähnlichen Prinzipien besteht (Roth und Ryba 2016, 324ff.). Der Unterschied liegt eher in der Rigidität zu verändernder Muster, die in klinischen Fragestellungen einen höheren Grad aufweist, was entsprechend mit einer höheren Beziehungsintensität in der Veränderungsbeziehung beantwortet werden muss. Dies lässt sich in der Praxis der Transaktionsanalyse sehr gut beobachten, weil sie sowohl im therapeutischen Feld als auch im Coaching Anwendung findet. Insgesamt sticht beim Coaching ein interdisziplinär und multiperspektiv geprägtes Arbeiten hervor. Elemente aus der Pädagogik, insbesondere der Erwachsenenbildung, der Organisationswissenschaft, der Soziologie, der Philosophie und weiterer Gebiete fließen in die Kompetenzausübung des Coaches ein. Coaching lässt sich zudem als eine Profession konzipieren: „Professionen zeichnen sich dadurch aus, dass sie auf zentrale Bezüge des Menschen ausgerichtet sind." (DBVCKompendium 2012, S. 12). Wie andere klassische Professionen wie Seelsorger, Arzt, Jurist oder Lehrer wäre hier die These, dass der/die Coach für das gesellschaftlich-wirtschaftlichen Gefüge eine ganz bestimmte Rolle und Dienstleistung übernimmt, mehr dazu weiter unten.

So macht es für die aktuelle Praxis noch für die Theorie weniger Sinn, im ehemaligen Ursprung der psychotherapeutischen Schulen und der Literatur der Psychotherapiegründerväter und -mütter zu suchen, um die heutige Qualität einer Methode für Coaching zu eruieren. Viel mehr macht es Sinn zu schauen, wie der aktuelle Stand einer Methode in Theorie und Praxis heute die allgemeinen Grundanforderungen für ein zeitgerechtes Coachingverfahren abbildet. Daher bleibt hier für die Transaktionsanalyse zu prüfen, wie der aktuelle Stand der Transaktionsanalyse sich heute im Coaching in eine integrierte Beratungsform einfügen lässt.

\section{Transaktionsanalyse - Wissenschaft und Fachliteratur}

Seit der Auflegung der ersten TA-Zeitschrift, dem Transactional Analysis Bulletin 1962 wurden mehr als 2000 qualitative Studien zur TA verfasst. 340 Artikel davon enthalten empirische und quantitative Forschung, 110 sind kontrollierte Wirksamkeitsstudien und wenigstens 33 erfüllen die Kriterien der Randomized Controlled Trials (RCT). Diese Zahlen haben 2020 die Vertreter der spanischen TA-Assoziation APPHAT (Asociacion de Profesionales de Psicologica Humanista y Analisis Transaccional) zusammengetragen, als sie vom spanischen Gesundheitsministerium aufgefordert wurden, den wissenschaftlichen Nachweis von TA darzustellen (APPHAT 2021).
Zwei Untersuchungen aus dem therapeutischen Sektor und zwei aus dem Coaching- bzw. Organisationsbereich seien hier kurz vorgestellt. Vos und van Rijn (2021) konnten in einer Meta-Studie auch zum Vergleich von TA und anderen humanistischen Ansätzen zeigen, dass TA sich in ihrem Wirksamkeitsgrad nicht von anderen unterscheidet (vgl. auch Van Rijn und Wild 2016). Horn et al. (2015) zeigten in einer Untersuchung, die auch vom Gutachten zur Humanistischen Psychotherapie angenommen wurde (Wissenschaftlicher Beirat Psychotherapie, Deutsches Ärzteblatt 2018), dass TA in der Behandlung von Persönlichkeitsstörungen hochwirksam ist. Eine eigene Untersuchung im Organisationsbereich zeigte, dass Teilnehmer an einer systemisch-transaktionsanalytischen Coachinggruppe nach einiger Zeit seitens der Organisation von einer vom Coaching völlig unabhängige Stelle allein aufgrund ihres sichtbaren Führungsverhaltens mehr Verantwortung übertragen bekommen (Mohr 2014). Einen ganz anderen Bereich betraf eine Studie zur Organisationsentwicklung, in der das Konzept der inneren Grenzlinien in Organisationen (Berne 1979) in Bezug auf die Mitgliedschaft von Frauen in Aufsichtsräten betrachtet wurde. Dabei zeigte sich, dass der Anteil von Frauen in Aufsichtsräten zwei Resultatsgrößen in Unternehmen, die Umsatzrentabilität und den Mitarbeiterzuwachs nicht signifikant verändert (Mohr 2019). Frauen und Männer verhalten sich in ihren Rollen in Unternehmen ähnlich. Dieser Rollenkompetenznachweis von Frauen im Management belegt auch den Nutzen der mittlerweile festen Genderquote von mindestens $30 \%$ in Aufsichtsräten.

Es gibt direkt auf transaktionsanalytisches Coaching bezogene Fachliteratur (z. B. Schmid 2003; Mohr 2008, 2010, 2013, 2017; Hay 2007; Dehner und Dehner 2013; Pratt 2021). Bernd Schmid gebührt das Verdienst, die Transaktionsanalyse in Verbindung mit der systemischen Betrachtung als Methode für das Coaching in Organisationen fruchtbar gemacht zu haben. Sein noch im Eigenverlag 1986 erschienenes Buch ,Systemische Transaktionsanalyse" bildete den Grundstock von transaktionsanalytischer Arbeit in Organisationen. Zwar hatte Berne schon ein Buch zur Transaktionsanalyse in Zusammenhang mit Organisationen veröffentlicht (Berne 1963/1979). Dies bestand allerdings aus einem Nebeneinanderstellung verschiedener methodischer Elemente ohne eine zusammenhängende Theorie. Dies wurde dann später von Fox (1975) versucht. Es blieb aber weiter wenig konsistent. Als interessante Ansätze sind hier auch die beiden österreichischen Transaktionsanalytiker Werner Vogelbauer und Hans-Georg Hauser zu erwähnen, die schon in den 1990ern Arbeiten zur Anwendung von TA im Organisationskontext beschrieben (Vogelauer 1991; Hauser 1991). Ein bemerkenswerterer Artikel kam von Rolf Balling, der mit „Motivation, Manipulation und Motipulation" deutlich gegen merkwürdige Vorstellungen in Firmen Stellung bezog und eine eindeutige Eine- 
Augen-Höhe-Beziehung mit Mitarbeitern propagierte (Balling 1989). Ute und Heinrich Hagehülsmann (1998) haben mit „Im Spannungsfeld der Organisation“ ein gutes, praxisorientiertes Anwendungswerk der Transaktionsanalyse im Organisationsbereich verfasst. Das Buch „Positive Führung" von Schulze und Sejkora (2015) thematisierte dann insbesondere Burn out und Resilienz in der Arbeitswelt.

Die Engländerin Julie Hay, die schon in ihrem Buch „Transactional Analysis for Trainers“ (Hay 1995) die TA für das Training in Firmen beschrieben hatte, unterscheidet in „Reflective Practice and Supervision for Coaches“ zwischen ,traditional coaching“ und „developmental coaching“, um den Übergang von einer auf Aufarbeitung früherer Beeinträchtigungen fokussierten Herangehensweise zu einer positiv-ressourcenorientierten Betrachtung anzuzeigen. Mittlerweile sind neben der systemischen Transaktionsanalyse (Schmid 1986, 1994; Mohr 2000, 2020) eine Reihe weiterer Sparten entstanden: „Co-creative TA“ (Tudor und Summers 2014), „Mindfulness-Based Transactional Analysis“ (Žvelc et al. 2011), ressourcenorientierte TA (Kessel et al. 2020), „Contextual TA“ (Sedgwick 2021) entstanden. Transaktionsanalyse zeigt darin die offene und gute Verbindungsmöglichkeit zu neu auftretenden Themen in den Beratungskontexten. Nicht zu vergessen ist für das Coaching auch die vielfältige transaktionsanalytische Literatur zur Supervision, die im transaktionsanalytischen Qualifizierungssystem eine große Rolle spielt und weil die methodische Überschneidung von Supervision und Coaching doch recht hoch ist. Die Beiträge von Schneider (2000) bis hin zum Sammelband von Brunner und Sell (2021) geben hervorragend Auskunft.

Transaktionsanalyse besteht heute ökonomisch ausgedrückt aus zwei Produktgruppen, einerseits der Theorie und ihrer Anwendung - dazu weiter unten - andererseits auch aus ihrem spezifischen, in einem über 60 Jahre international erprobten und ständig weiterentwickelten Qualifizierungssystem. Verhilft nun das transaktionsanalytische Qualifizierungssystem zu einer Professionalisierung, die den Anforderungen einer Profession gerecht werden? Das Kompendium „Coaching als Profession“ des Deutschen Bundesverbandes Coaching zählt dazu zehn traditionelle Merkmale einer Profession auf (DBVC-Kompendium 2012, S. 12f.). Ähnlich wie dem Coaching allgemein fehlen davon der Transaktionsanalyse in Deutschland - anders als in Großbritannien oder in Italien beispielsweise - staatliche Lizenz und gesellschaftliches Mandat, was zwei der Merkmale einer Profession sind (ebenda, S. 14). Jedoch stellt die Transaktionsanalyse etwas, das allgemeingültig im Coaching erst einmal nicht erfüllt ist, sehr genau zur Verfügung, nämlich einen differenzierten und wissenschaftlich überprüften standardisierten „Karrieregang“ (ebenda, S. 14), zumindest was die Qualifizierung anbelangt (siehe dazu Handbuch der Europäischen Gesellschaft für Transaktionsanaly- se: https://eatanews.org/exams/\#handbook oder das Handbook der International Transactional Analysis Association, www.itaaorg.com). Insofern hat sie gute Grundlagen, die Profession Coaching mit ihren Ansätzen zu unterstützen.

\section{Vorschlag für eine metatheoretische Perspektive der Transaktionsanalyse}

Die Transaktionsanalyse wurde schon mit dem Vorwurf konfrontiert, nur ein Sammelsurium von Handwerkszeugen zu sein und ,keine fundierte Theorie“ zu besitzen (Roth und Ryba 2016, S. 41). Dies soll im Folgenden betrachtet werden. Schreyögg (2012, S. 107 ff.) stellt vier Anforderungen an die Wissensstruktur eines Coachingmodells: Vorhandensein eines Meta-Modells, einer Theorie-Ebene, grundlegender methodischer Anweisungen und einer Praxeologie. Die vier Perspektiven sind noch um die wissenschaftliche Basis des Konzeptes zu erweitern, die man zwischen Metatheorie und Einzeltheorien anordnen kann. Ist bei einem Verfahren eine Metatheorie vorhanden und welche erkenntnistheoretischen und anthropologische Prämissen gibt es? Wie der Name schon sagt, ist Transaktionsanalyse in erster Linie eine Theorie des Austausches, also des „Zwischen“ von Menschen. Ähnlich der Philosophie Martin Bubers gilt für die Entwicklung des kleinen Menschen wie auch für erwachsene Beziehungen „Über das Du zum Ich werden“ (Buber 1983, S. 37). Die Beziehung zwischen Menschen ist dabei für die Transaktionsanalyse durch radikale Wertschätzung geprägt. Die wichtigste Triebfeder des Menschen wird in der Orientierung auf Anerkennung, Kontakt und Zugehörigkeit gesehen. Anerkennende und resonante Beziehungen, wie sie auch in der sozialphilosophischen Forschung durch Honneth (1992) und Rosa (2016) postuliert werden, stehen im Zielsystem der TA. Die anthropologische Annahme ist, dass die tolerante und grundlegend wertschätzende Haltung gegenüber dem eigenen Gedeihen wie auch dem Zusammenleben und Zusammenwirken der Menschen untereinander von entscheidendem Nutzen sind. Der Mensch ist auf der Basis seiner Beziehungsorientiertheit an Entwicklung und Wachstum interessiert. Die TA fußt hier auf der Rousseau'schen Philosophie des positiven Menschenbildes. Die Annahme ist die eines grundlegenden Willens, sich positiv in die Welt einzubringen und darin seine "Ressourcen“ zu realisieren (Kessel et al. 2020). Eine zweite These ist: Der Mensch möchte darin eine autonome, im Sinne von nicht gegen seinen Willen fremdbestimmte, sondern selbstbestimmte Person sein. Dies wurde in den Anfängen sehr stark als Auseinandersetzung mit der elterlichen Prägung angesehen, heute wäre es durchaus auch gerade bei Heranwachsenden vielleicht eine Autonomie von Beeinflussung durch die so genannten sozialen Netzwerke. Die innerpsychische Struktur des Menschen ist nach der transaktions- 
analytischen Theorie eine Abbildung seiner bisherigen Beziehungserfahrungen. Zeigen sich darin Geborgenheit und positive Kooperationserfahrungen mit anderen, so zeigt sich das auch im weiteren Leben. Die zugespitzte Formulierung von Harris des „Ich bin ok, Du bist ok“ reicht viel weiter als auf zwei, sondern auch auf die Bezugnahme zu allen anderen Menschen, zu Institutionen und Organisationen, zur Gesellschaft, zur Natur (Mountain und Davidson 2015). Heute geht es um die relevanten aktuellen Kontexte des Menschen (Mohr 2020; Sedgwick 2021). Vieles lässt sich nicht allein durch Kindheitserfahrungen erklären. Entsprechend stellt auch die Weiterentwicklung ethischer Positionen für die professionelle Arbeit (vgl. Hallstein 2021) heute einen wesentlichen Aspekt der TA dar, die die weitreichenden Konsequenzen und die unterschiedlichen Kontextbezüge des Handelns betrachtet.

TA ist „entwicklungsoptimistisch“, aber auch „realistisch" (Mohr 2008). Vor allem Letzteres beruht darauf, dass die TA-Theorie sich durch die Arbeit in sehr anspruchsvollen Feldern (Gefängnis, Psychiatrie) und auch für sehr kompetitive Kontexte wie der Wirtschaft weiterentwickelt hat und dadurch beispielsweise nicht blind ist für das destruktive Potenzial, das in manchen Kontexten aufkeimt. Gerade die Arbeit mit rigiden Mustern hat die Notwendigkeit der komplexen Beziehungsgestaltung aufgezeigt, in der Übertragungs- und Gegenübertragungsaspekte eine große Rolle spielen und beraterisch genützt werden (Sills und Hargarden 2003; Tudor und Summers 2014). Auf diesem differenziert systemisch-beziehungsorientierten Hintergrund geschieht heute transaktionsanalytisches Coaching, um Entwicklung und Wachstum zu erzielen.

Erkenntnistheoretische Kernpunkte der Transaktionsanalyse sind Bewusstheit, Flexibilität und Bezogenheit auf Menschen und Themen. Bewusstheit impliziert eine absolute Transparenz dessen, was im Coaching passiert für den Klienten. Es ist eine autonome Bewusstheit, die die TA beispielsweise von aus der Hypnotherapie stammenden Verfahren wie etwa NLP (Neuro-Linguistisches Programmieren) unterscheidet. Interessanterweise propagiert heute Gunther Schmidt, der auch viel mit Transaktionsanalytikern in Kontakt stand, in seinem hypnosystemischen Ansatz ebenfalls vollständige Transparenz des Vorgehens. Von Seiten der Transaktionsanalyse ist eine absolute Aufklärung und ein „Vertrag“ nötig, was eine transparente, wechselseitige Übereinkunft zum Vorgehen bedeutet. Flexibilität bedeutet ein Erhöhen der Wahlmöglichkeiten des Handelns, des Bewertens und damit auch des Fühlens zu einer Situation. Bezogenheit ist das, was der Soziologe und Sozialphilosoph Hartmut Rosa (2016) sehr differenziert als „Resonanz“ definiert. Resonanz auf den drei Achsen Beziehungen zu Menschen, Tätigkeiten und Objekte sowie auf große Themen wie Kultur, Musik oder Spiritualität bedeutet für Rosa eine klare und emotionale Reaktion,

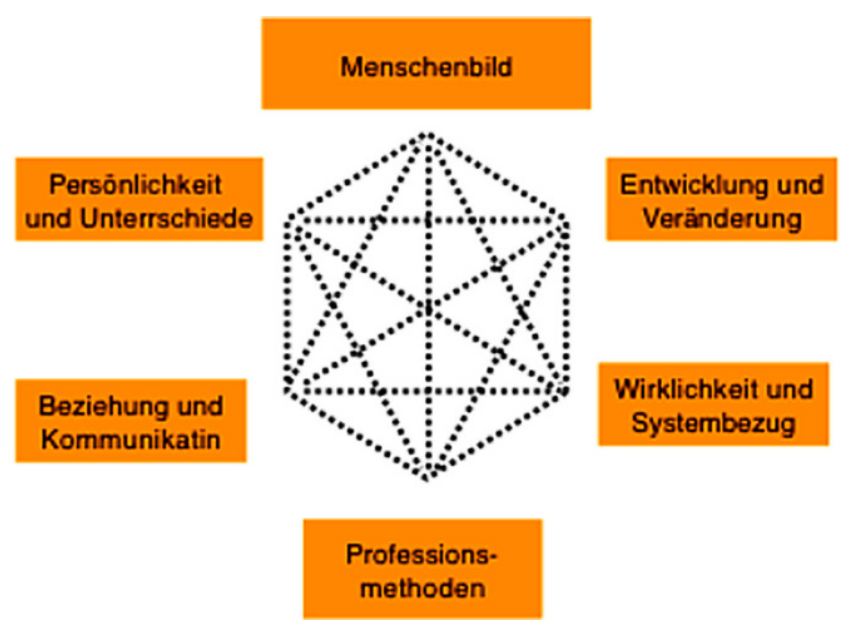

Abb. 1 Diamant der Professionalität (Mohr 2020)

die mit dem eigenen Wesen zu tun hat, intrinsisch Selbstwirksamkeit erleben lässt und die einen ein kleines Stück transformiert, also vorankommen lässt (Rosa 2016, S. 298). Die Trias Bewusstheit, Flexibilität und Bezogenheit wird in der TA auch mit „Autonomie“ bezeichnet (Mohr 2020), eine Begriffsbildung, die ähnlich wie der gerade genutzte Resonanzbegriff über die umgangssprachliche Verwendung hinausgeht und im theoretischen Rahmen mit seinen spezifischen Definitionsmerkmalen zu nutzen ist.

\section{Zentrale Einzelkonzepte der Transaktionsanalyse}

Um die Begegnungen und das Zusammenwirken der Menschen in guter Weise zu entwickeln, stellt die Transaktionsanalyse unter dem Schirm der beziehungsorientierten Metatheorie eine ganze Reihe von einzelnen theoretischen Modellen zur Verfügung. Hier gibt es wesentliche Aussagen zu Menschenbild, Persönlichkeit und Unterschiedlichkeit, Beziehung und Kommunikation, System- bzw. Kontextbezug, zu Entwicklung und Veränderung sowie zu Professionsmethoden.

Viele Einzelkonzepte sind in der Transaktionsanalyse mit interessanten Metaphern („Parent ego State“ (Eltern-Ichzustand), „Child ego State“ (Kind-Ichzustand), „Games“ (Spiele), „Strokes“ (Streicheleinheiten), ...), aber aus Theoriesicht etwas unglücklich bezeichnet. Sie sind dadurch für viele Menschen zwar erst einmal didaktisch günstig vermittelbar, es braucht zur theoretischen Einordnung allerdings dann eine zweite Ebene von Prägnanz und Schärfe. Ein Beispiel dazu ist die Ichzustandstheorie, die durch die Forschungsarbeit von Temple (2002) erst wissenschaftlich ohne Metaphern wie „Eltern“ oder „Kind“ formuliert wurde und damit überprüfbar war. In der folgenden Darstellung werden die Modelle, wie sie Aussagen zu sechs Fragestel- 
lungen machen, dargestellt: Menschenbild, Persönlichkeit und Unterschiede, Beziehung und Kommunikation, Entwicklung und Veränderung, Wirklichkeit und Systembezug sowie Professionsmethoden (Abb. 1).

\subsection{Das Menschenbild}

Das Menschenbild der Transaktionsanalyse ist entwicklungsoptimistisch, realistisch, systemisch und emanzipatorisch. Drei Grundbedürfnisse nach Berne (Anerkennung, Stimulation und Struktur) sowie drei Triebkräfte (Überleben, Gestaltung, Ruhe) nach English (1972a, b) beschreiben ein Vorstellungsbild über den Menschen. Das Element Struktur findet sich in der TA häufig wieder. Das Zielbild der Bezogenheit und der Autonomie rundet dies ab. Neuere TA-Autoren wie etwa Kessel et al. (2020) betonen das Positive in der Transaktionsanalyse neu und nennen ihre Herangehensweise entsprechend „Ressourcenorientierte Transaktionsanalyse“. Wie oben in der Metatheorie beschrieben, fußt TA als humanistisch-psychologischer Ansatz letztlich auf Rousseau'schem Gedankengut, das den Menschen als von Natur her positiv ausgestattet postuliert. Umfeldeinfüsse, etwa durch die Eltern, tragen im ursprünglichen TA-Ansatz zu Einschränkungen bei. Der systemischtransaktionsanalytische Ansatz ist in diesem Punkt etwas neutraler, sieht auf epigenetischer Theoriebasis auch von vorne herein die Potenziale von Menschen, sehr destruktiv handeln zu können. Dafür immer die Elternbeziehung verantwortlich zu machen, erscheint nach heutigem Wissen in vielen Fällen auch nicht plausibel.

\subsection{Das Persönlichkeitskonzept}

Hier arbeitet der transaktionsanalytische Coach mit vorwiegend zwei Perspektiven. Eine ist die Perspektive des $\mathrm{Zu}$ sammenwirkens verschiedener Strebungen, Stimmen, Teilpersönlichkeiten (Ichzustände) in der Persönlichkeit, deren Einklang für erstrebenswert gehalten wird. Hier unterscheidet sich die Transaktionsanalyse nach Peichl (2019) von anderen Ansätzen dadurch, dass sie für Veränderungsprozesse keine Tranceerfahrung des Klienten für nötig hält. Die Transaktionsanalyse ist sicher die Methode, die den Teile-Ansatz der Persönlichkeit am meisten gefördert hat, weil Berne ein direkter Schüler Paul Federns war, der als Begründer dieses Ansatzes gelten kann (Federn 1953). Interessanterweise bezieht sich auch Friedemann Schulz von Thun, der den Grundgedanken in seinem „Inneres Team“Ansatz im deutschsprachigen Raum sehr populär gemacht hat, auf Transaktionsanalyse, da er auch Ausbildung in TA genossen hat. Mittlerweile ist im deutschsprachigen Raum der Ego-state-Therapie-Ansatz, der sich an die Hypnotherapie anlehnt (Fritzsche und Hartmann 2010: Peichl 2019) ebenso weit verbreitet.
Eine zweite Perspektive ist die des Lebensnarrativs (Skript). Dieses zentral geisteswissenschaftlich orientierte Konzept eines durch frühe Lernerfahrungen entstehenden Lebensdrehbuches wird auch in vielen Coachingübungen etwa der Lebensflussübung (Höher 2020, S. 239) genutzt. Die Idee beim Lebensnarrativ ist, dass der Mensch für sein Leben eine unbewusste Geschichte entwickelt, nach der er sein Leben steuert. Dies gilt in dem Sinne, welche Themen ihm wichtig sind, welche Personenkonstellationen und -typen er im Leben in Arbeit und Liebe sucht. Diese rein geisteswissenschaftlich begründbare Phänomen einer inhaltlich zusammengefügten Geschichte ist ein spannender Ansatz, der der hirnorganischen Forschung noch einige Rätsel stellt.

\subsection{Beziehung und Kommunikation}

Die Beziehung ist der zentrale Angelpunkt. „Vom Lebensplan zum Beziehungsraum" beschreiben Sejkora und Schulze (2017) die Bewegungsrichtung in einer transaktionsanalytischen Beratung. Korpiun (2020) spricht vom Ende der Individuumszentriertheit. Wenn man sich der Betrachtung von der Einzelperson löst, werden genau die durch die Begegnung von Menschen entstehenden besonderen Phänomene interessant. Übertragungs- und Gegenübertragungsprozesse zwischen Klient und Coach, in denen jeweils eigene Themen durch Projektion beim anderen lokalisiert werden, rücken in den Betrachtungsfokus. Ebenso interessant sind so genannte Parallelprozesse, dass über mehrere Ebenen selbst in seiner Supervision vom Coach ein Thema in derselben Qualität eingebracht wird, wie es zwischen dem Coach und seinem Klienten sich ereignete.

\subsection{Wirklichkeit und Kontext}

Die Ressourcen- und Resilienzorientierung sind in der Gestaltung der inneren Wirklichkeit zentrale Punkte. Diese sehr systemisch-konstruktivistisch ausgelegte Konzeption arbeitet mit dem so genannten Bezugsrahmen, der aktuellen Sicht eines Menschen auf sich selbst und seine Beziehungen zu anderen und zur Welt, die mittels Wertung und Abwertung von Phänomenen der Wirklichkeit aufrecht erhalten wird. Dies korrespondiert mit einem inneren Kontext, der biografischen Erfahrung des Skriptes. Sedgwick (2021) nennt diese Perspektive in seiner kontextbezogenen TA vertikal. Gleichermaßen und aus seiner Sicht sogar oft bedeutsamer bezeichnet er die horizontale Perspektive, die aktuell geltende Kontexte wie etwa das Milieu oder die gesellschaftliche Schicht eines Mensch mehr in der Vordergrund rückt.

Im Bereich der Wirklickeitskonstruktion und Kontextbezogenheit liegen mittlerweile Theorien für das Einzelsystem Person genauso wie für Organisationen vor. Die Ein- 
zelperson wird vor allem in ihren Rollenwelten betrachtet. Privatwelt, Professionswelt und Organisationswelt (Schmid 2003), sowie Gemeinwesenwelt und Konsumwelt (Mohr 2020) beeinflussen das was aus der Persönlichkeitsstruktur eines Menschen kommt, sehr deutlich. Genauso ist die Tradition der Modellentwicklung für Organisationssysteme (Balling 2005; Mohr 2006). Auch erste Ansätze systemisch-transaktionsanalytischer Modelle für die Volkswirtschaft insgesamt (Mohr 2015) und Politik (Mohr 2020) runden dies $a b$.

\subsection{Entwicklung und Veränderung}

Entwicklung und Veränderung kann durch Transaktionsanalyse auf vielerlei Hinsicht geschehen. Im Gutachten zur Humanistischen Psychologie wird die Veränderungstheorie der TA mit der so genannten Enttrübung beschrieben. Die bedeutet, dass für eine aktuelle Lösung und Entwicklung notwendige Kompetenzen (Erwachsenen-Ich) von alten einschränkenden Mustern (Eltern-Ich und Kind-Ich) befreit (,enttrübt") werden und so der aktuellen Situation voll zu Gute kommen können. Die alten Muster können frühe eigene (Kind-Ich) wie auch von anderen übernommene Haltungen (Eltern-Ich) beinhalten. Entwicklung und Veränderung bezieht sich gerade im Coaching aber auch auf Neulernen im Rahmen des Bezugsrahmens wie auch der Rollen. Genauso kann Veränderung in Systemen durch den Coach, wenn er das System als Klienten adressiert, auch auf der Ebene der Dynamiken eines Systems (z. B. Kommunikationsmuster, Problemlösemuster) erfolgen, indem beispielsweise Formen wie kollegiale Beratung in der Problemlösung eingeführt werden. Somit liegen aufgrund der Modellvielfalt auch sehr vielfältige Ansatzpunkte für Veränderung vor.

\subsection{Professions- und Interventionsmethoden}

Die anfängliche revolutionäre Vorgehensweise von Berne, „mit“ dem Patienten in einer Therapiekonferenz zu sprechen und ,nicht über ihn“ (Berne 2005). fand später in den systemischen Ansätzen ihre Fortsetzung. Auf der Basis radikalen Respekts und der ethischen Grundhaltung geht der Transaktionsanalytiker auch in die Beziehung mit dem Coachee auf eine Augenhöhe. Es gilt, gemeinsam in einem kokreativen Prozess (Tudor und Summers 2014) nach Lösungen für das System des Klienten zu suchen. Grundlage allen professionellen Arbeiten in der TA ist der „Vertrag“. Diese wechselseitige Übereinkunft in einem Arbeitsbündnis hat sich mittlerweile in allen Coachingansätzen durchgesetzt. Für manche ist der Begriff „Vertrag“ sprachlich etwas ungewöhnlich. Allerdings hat er eine deutlich mehr emanzipatorische Konnotation als der in der systemischen Theorie präferierte Begriff „Auftragsklärung“, der im Coach oft implizit zu einem auftragserfüllenden Dienstleister und nicht die zu einer Entwicklung stimulierenden Profession sieht. Entsprechende Einordnung in die standardisierten Einkaufsprogramme von Firmen fördern dies zusätzlich. Insgesamt bleibt diese Begriffsfrage aber auch Geschmacksache. TA ist darüberhinaus wenig bestimmend bezüglich der methodischen Vorgehensweise. Der TAler spricht Umgangssprache, nutzt die Modelle zur Illustration, importiert aber auch gerne praktische Methodik aus anderen Verfahren.

In der praktischen Intervention lassen sich sehr gut die drei Bezugnahmen auf den Coachee nach Erskine et al. (1999) und die von Berne (1961/2005) schon beschrieben Grundinterventionen verbinden. Inquiry bedeutet das Einnehmen einer erkundenden Haltung, Attunement zeigt sich im eng bei Klienten, etwa auch seiner Sprache, bleiben. Im Involvement zeigt sich der Coach auch mit seinen lebendigen Reaktionen auf den Coachee. Die acht in der Kopfzeile aufgeführten Interventionen sind die klassischen von Berne (1961/2005). Beide Perspektiven, die Haltungsperspektiven von Erskine und die praktischen von Berne, ergänzen sie sich gut. Wie oben schon angedeutet, ist die Transaktionsanalyse aufgrund ihrer Multiperspektivität auf aktuelles Verhalten, biografische Zusammenhänge und systemische Kontexte ebenso offen für die Kombination mit anderen Konzepte wie etwa Aufstellungen, körperorientierten Methoden oder Bilderarbeit wie im Zürcher Ressourcenmodell, um hier nur einige Beispiele zu nennen (Tab. 1).

Der Unterschied der Transaktionsanalyse zu anderen humanistisch-psychologischen Ansätzen zeigt sich in der Deutung von Situationen mithilfe der Einzeltheoriemodelle der TA. Darin steckt ein besonderer Reichtum der Transaktionsanalyse. Diese Musterkonstruktionen für psychische Situationen (Mohr 2012) werden oft als "die“ Transaktionsanalyse beschrieben, also die Konzepte Ichzustände, Transaktionen, Skript und psychologische Spiele. Dies ist kritisch anzumerken. Mit dem Überspringen der oben betrachteten grundlegenden erkenntnistheoretischen und anthropologischen Ebene haben Transaktionsanalytiker manchmal selbst beigetragen, ihre Theorie und deren

Tab. 1 Interventionstabelle

\begin{tabular}{|c|c|c|c|c|c|c|c|c|}
\hline & Befragung & Hervorhebung & Erklärung & Visualisierung & Konfrontation & Bestätigung & Deutung & Kristallisation \\
\hline \multicolumn{9}{|l|}{ Inquiry } \\
\hline \multicolumn{9}{|l|}{ Attunement } \\
\hline Involvement & & & & & & & & \\
\hline
\end{tabular}


wissenschaftliche Kommunizierbarkeit zu vernachlässigen, indem sie sich oft wie aus einer szeneinternen Sicht nur im Begriffsrahmen der Einzelmodelle bewegten und die Anknüpfung an andere Sprach- und Theoriewelten vernachlässigten (z. B. Cornell et al. 2016). Die zusätzlich sehr lange und gerade im Coachingfeld als ausgedehnt empfundene Ausbildung, die noch in der Tradition der Psychoanalyse stand, tat neben den eigenen Sprachbegrifflichkeiten ihr Übriges, um die Transaktionsanalyse von einem der vorderen Plätze in der Beratungs- und Therapielandschaft nach hinten zu verdrängen. Mittlerweile werden in der TA deutlich kürzere Ausbildungsgänge etwa für Coaching angeboten (Deutsche Gesellschaft für Transaktionsanalyse, www.dgta.de). Aber die Möglichkeit zur langen, sehr tiefen Ausbildung soll gerade die oben erwähnte so zentrale Beziehungskompetenz erzeugen und wird durch das umfassende Qualifizierungsangebot gewährleistet. Diese Lernerfahrungen sind in anderen Coachingweiterbildungen oft auf das Lernen on the Job, also auf nach der Ausbildung und auf die Nachausbildungssupervision vertagt.

Veränderungen entstehen in Einzel- oder Mehrpersonensystemen durch eine Irritation, ein Innehalten, eine Reflexion, oder eine Neuentscheidung. In der Nutzung dieser Maximen gehen die Transaktionsanalytiker sicher weiter als andere humanistische Ansätze wie etwa die klientenzentrierte Gesprächsführung nach Rogers. Aufgrund der Erfahrung, dass eine Intervention ein Klientensystem auch Aufmerksamkeit (Mohr 2013) erzeugen muss, konfrontiert der Transaktionsanalytiker auch, wobei Konfrontation klar als Aufzeigen von Widersprüchen beim Klienten (Berne 1961/2005) oder als Gegenüberstellen der eigenen Haltung zu einem Thema mit der des Klienten (Schiff et al. 1975) definiert ist. Entsprechend der Metatheorie der Veränderung nach Eidenschink (2016) sind Entscheidungen des Klienten wichtig. Dies korrespondiert mit dem Konzept der Neuentscheidung, das auch in der TA eine große Rolle spielt (Goulding und Goulding 2005). Die achte Interventionstechnik von Berne (2005) das ,vor die Entscheidung Stellen“ ist die Grundlage der Änderung bei einer Reaktionsweise.

Bis hierhin erst mal die überblicksartige Darstellung der metatheoretischen und einzeltheoretischen Überlegungen zur Transaktionsanalyse. Im Folgenden werden an einem Fallbeispiel über ein Coaching in einer Organisation noch einmal praktische Vorgehensweisen dargestellt.

\section{Fallbeispiel Multilevel-Coaching}

Im Fallbeispiel geht es um ein modernes transaktionsanalytisch orientiertes Coaching der Werkleiter in einer britischen Fabrik eines deutschen Konzerns. Anlass war, das die Performance der britischen Fabrik so schlecht war, dass sie im Vergleich zu den anderen europäischen Firmen, die alle mit der gleichen Produktionsanlage ausgestattet waren, die „rote Laterne“ trug, also die schlechtesten Ergebnisse erzielte. Nachdem andere Lösungen wie zahllose Fachberatungen vor Ort durch deutsche Ingenieure und auch Führungstrainings für die britischen Werkleiter in Deutschland nicht die erhofften Verbesserungen gebracht hatten, fragte mich die Konzernleitung, ob ich als Coach für die zwei Werkleiter in einer ihrer Fabriken in Großbritannien zur Verfügung stünde. Ich selbst kannte die Firma nicht. Ein Kollege hatte mich empfohlen, da er wusste, dass ich im internationalen Kontext Erfahrung habe. Ich entschied mich nach der Auftragsklärung/Vertragsarbeit die Methoden Shadowing und Multilevelcoaching anzubieten, was vom Klientensystem angenommen wurde. Beim Shadowing geht der Coach in die Organisation, in diesem Falle eine Fabrik, hinein und interveniert unmittelbar. Multilevelcoaching bedeutet, dass alle Hierarchieebenen im Entwicklungsprozess in ihren spezifischen Rollen involviert sind. Was bedeutete das im Einzelnen? Hier einige Interventionsbeispiele:

\subsection{Vertragsarbeit}

Zunächst galt es mit der Konzernleitung, vertreten durch den Produktionsleiter, den beiden britischen Werkleitern und mir einen Dreiecksvertrag (English 1975) zu schließen. Nach Berne gilt es drei Vertragsebenen zu unterscheiden, die administrative, die professionelle und die psychologische (Berne 2005). Zunächst willigte der Produktionsleiter in meinen Vorschlag ein, dass ich zweimal drei Tage in die Fabrik gehe und die Werkleiter coache (Shadowcoaching). Die wichtigsten Partner im Coaching, die Coachees, waren jetzt noch nicht an Bord. Die beiden wurden dann per Video auf Großleinwand zugeschaltet, ich stelle mich vor und die beiden Werkleiter stellten mir noch einige Fragen. Dann fragte der Produktionsleiter die beiden, ob sie mit mir das Coaching angehen wollten. Sie antworteten in ihrem Dialekt etwas, das sich signifikant von „No“ unterschied. Ich fragte mich, ob ich damit einen Vertrag hatte. Eher noch nicht. In der Transaktionsanalyse ist eine Vertrag eine wechselseitige Übereinkunft über ein gemeinsames Vorgehen (Berne 2005). Er sollte unter maßgeblichen Beteiligung des Erwachsenen-Ichs beider Parteien geschlossen werden. Wie weit die beiden Coachees aus dem Erwachsenen-Ich, also autonom, ohne unangemessene innere Pressionen aus Abhängigkeiten heraus, sprachen, war natürlich überhaupt nicht klar. Eher war anzunehmen, dass sie aus einer angepassten Haltung ,ja“ sagten.

Interessanterweise ging oben angedeutete Vertragsarbeit - als kleiner Vorgriff - am Ende des zweiten Tag meines Aufenthaltes in der Fabrik weiter. Ich fragte die Werkleiter, wie sie denn mit mir zurecht kämen. Die Antwort war ,as we realized that you are on our side ...". So schön ein Kompliment für mein offensichtlich gezeigtes Involvement und 
Attunement (Erskine et al. 1999) ist, ich konnte auch dies so nicht stehen lassen. Ich konfrontierte (Berne 2005) die beiden, indem ich aufzeigte, dass ich mich über das Vertrauen freue, aber sowohl auf ihrer Seite als auch auf der Seite der Konzernleitung sei. Das war komplex, aber sie nahmen es. Einer der Kernpunkte der Transaktionsanalyse ist die Beziehung und darin die geäußerte Beziehungshaltung. Im Organisationskontext ist dies allerdings eine Mehrecksbeziehung zwischen menschlichen Beziehungspartnern und Sachen, Zielen und Aufgaben. Die Frage für den Coach ist daher, welche Haltung verkörpert er und zu welchem Bezugsrahmen (Schiff 1975) lädt der Coach ein. Nun war ich nicht der erste Berater in diesem System und ich hatte schon in meinen Vorgesprächen mit dem deutschen Produktionsleiter eruiert, welchen Ansatz man hier bisher hier gewählt hatte. Man hat sehr deutlich Existenz und Tragweite der vielen „Probleme“, auch in seiner ständigen ebensolchen Etikettierung, verdeutlicht.

\subsection{Die erste Transaktion}

In der TA wird die Bedeutung der ersten Transaktion herausgestellt. Sie wird auch im Buchtitel von Bernes letztem Buches noch einmal verdeutlicht: „What do you say after you say Hello?" Entsprechend entschied ich ich nach längerem Überlegen auf die Eingangstransaktion bei jeder Neubegegnung, Was ist das Problem?' ausdrücklich zu verzichten, sondern für, Worauf sind Sie stolz bei der Arbeit? Was würden Sie Ihrem kleinen Sohn/ihrer kleinen Tochter in der Fabrik zeigen?' zu fragen. Dies tat ich bei jedem in der Fabrik, mit dem ich ins Gespräch kam: Werkleiter, Schichtführer, Maschinenarbeiter, Schlosser, Elektriker, Bürokräfte. Dies ergab interessante Antworten und vor allem ein anderes Klima als ,Was ist das Problem?‘.

\subsection{Interventionen}

Was tat ich nun den ganzen Tag in der Fabrik? Morgens ließ ich mir von den beiden Werkleitern ihre Vorhaben für den Tag schildern: Fabrikrundgänge, Gespräche mit einzelnen Mitarbeitern, Teilnahme an der Teambesprechung der Schlosser und Elektriker und dann der Schichtbesprechung der Arbeiter nach der Frühschicht (6-14 Uhr) um 14.00 Uhr. Ich begleitete die beiden Werkleiter bei ihrer täglichen Arbeit und intervenierte sehr unterschiedlich zu ihrem Tun.

Bevor ich auf konkrete Interventionsbeispiele eingehe, noch der Hinweis auf eine Vorbereitungsarbeit, die ich den Werkleitern vor meinem Eintreffen in der Fabrik zugemutet hatte. Ich gab ihnen den Fragebogen zur Systemischen Organisationsanalyse zum Ausfüllen. Dieser erfasst zehn Dynamiken zur Qualität einer Organisation (Mohr 2006, 2010). So kam zusammen mit Explorationsinterviews der von mir entwickelte Fragebogen SCISOA zum Einsatz. Damit können relevante zehn Systemdynamiken (Aufmerksamkeitsfokus, Rollen, Systembeziehungen, Kommunikationsmuster, Problemlösemuster, Erfolgsmister, Gleichgewichte, Rekursivität, äußere und innere Pulsation) ermittelt werden. In den zentralen Dimensionen Rollen und Systembeziehungen kamen unterdurchschnittliche Werte zum Vorschein. Damit zeigten diese Dimensionen das größte Potenzial für Verbesserungen.

Eine genaue Exploration erbrachte dann: Bei den vier im Konzern und in der Fabrik beteiligten Ebenen waren die unterschiedlichen Aufgaben in der vertikalen Teamarbeit nicht klar. Kommunikation und Erfolgsdefinition waren gut entwickelt. Man kommunizierte viel und direkt. Es war aber aufgrund der unklaren Rollenbeziehungen nicht unbedingt zielführend. Bezüglich der Erfolgsdynamik gab es klare Kriterien: die Produktionszahl und die Qualität der Produkte, Letztere gemessen anhand der Kundenreklamationen. Die Dimension ,Rollen und Beziehung ' ließen sich sehr gut am Beispiel der Zuordnung von Verantwortung für bestimmte Aufgaben fokussieren. Die Verantwortungen waren recht ungeklärt. Wer hatte Verantwortung für was? Wie war sie auf die verschiedenen Rollenträger Werkleiter, Schichtführer, Schlosser, Elektriker und Arbeiter aufgeteilt? In meiner Verhaltensbeobachtung vor Ort bestätigte sich dieser Befund. Ein Beispiel war der sichtbare Ordnungszustand im Werk. Es wurde hart und viel gearbeitet, im 24-Stunden-Betrieb in drei Schichten. Getreu dem Spruch „Wo gehobelt wird, da fallen Späne“ gab es überall Stellen in der Fabrik, wo kleine Metall- oder Holzteile herumlagen oder in Ecken des Werkes wurden nicht gebrauchte Materialien einfach übereinander gelegt. Dies war durchaus nicht günstig, weil durch die Fahrzeuge im Werk, etwa die Gabelstapler oder auch die großen LKWs, die die fertige Ware abholten, die Teile auch umhergeschleudert werden konnten, was sowohl Sicherheitsprobleme als auch immer wieder Verfahrensprobleme bei den durch Lichtschranken gesteuerten Förderbändern mit sich bringen konnte.

Nun eine Intervention zur Rollenthematik in diesem Zusammenhang: Wayne (Name geändert), einen der Werkleiter begleitete ich bei seinen Fabrikrundgängen. Da lag dann auf einmal ein etwa $15 \mathrm{~cm}$ langes Metallteil vor uns auf dem Boden. Wayne hob es auf und legte es an einen sicheren Ort. Ähnliches passierte bei dem Rundgang mehrere Male. Ich fragte ihn, ob das seine Aufgabe sei. Er sagte, nein, das sei Aufgabe der Arbeiter. Ich fragte ihn, warum er es denn gerade aufhebe. Er antworte, er käme ja ohnehin vorbei, sei ja dann sowieso da. Außerdem würde er so ein gutes Vorbild abgeben. Gefragt, ob das denn wirksam sei, musste er verneinen. Ich fragte ihn, ob er etwas versuchen wolle, was die Verantwortung deutlicher mache. Als er das bejahte, schlug ich ihm vor, den Schichtführer per headset anzurufen - in der Fabrik war es sehr laut - diesen einen 
Arbeiter beauftragen zu lassen, der für diesen Sektor zuständig war, um das Teil aufzuheben und an einen sicheren Platz zu bringen. Zugeben solche Interventionen machten mich in der Fabrik nicht nur beliebt. Aber die Verantwortungshandhabung in diesen Situationen wurde sehr schnell sichtlich besser.

\subsection{Bedürfnis nach Stimulation und Aufmerksamkeitsfokussierung (Illustration I)}

Eine weitere Intervention bezog sich auf die Dimension „Problemlösung“. Mir war aufgefallen, dass an den Stellwänden im Betrieb negative Bilder von ungeordneten Situationen aufgehängt waren. Eine Analyse der Aufmerksamkeitszentrierung ergab, dass man bisher versucht hatte, mit Veranschaulichung der Probleme zu Lösungen zu motivieren. Dies erschien mir nicht ressourcenorientiert. In einem längeren Beratungsprozess arbeiteten wir heraus, dass stattdessen positive (Vor-)Bilder ausprobiert werden könnten.

Ihre erste Idee war, Bilder aus der deutschen Musterfabrik zu nehmen. Die Deutschen seien ja auch bekannt für ihre Ordnung, sage Wayne. Irgendetwas gefiel mir daran nicht. In der Transaktionsanalyse spielen unterschwellige Botschaften und auch Intuition eine große Rolle. Die „deutschen Oberlehrer" sollten Bilder liefern. Was würde das bewirken? Sie hätten natürlich auch selbst positive produzieren können. Aber ich wollte schnell - vielleicht mein Beeil-Dich-Antreiber - sein und zügige Lösungsmodalität fördern. Da fiel mir ein, dass ich den Manager der polnischen und der tschechischen Fabrik im Vorgespräch in Deutschland kennengelernt hatte und wir uns gut verstanden hatten. Polen und Tschechen als alte Alliierte der Briten erschienen mir geeignet. Ich fragte deshalb Robin, den zweiten Werkleiter, ob er diesen Mann kenne und ihn nach positiven Bildern aus der polnischen und tschechischen $\mathrm{Fa}-$ brik fragen könne. Innerhalb eines Tages hatten wir die Bilder von den „Schwesterfabriken“. Veranschaulichungen haben eine hohe Motivationskraft. Daher sollten nicht negative, sondern positive Beispiele veranschaulicht werden. Das habe ich getan. Die Bilder von anderen Standorten zeigten eine aufgeräumte, geordnete Fabrik. Zusätzlich konnte ich diese Bilder noch für eine weitere Intervention nutzen, die weiter unten beschrieben ist.

\subsection{Mediation der Schlosser und Elektriker}

Ein Problem waren häufige Störfälle bei einer Maschine. In allen Räumen der Fabrik ragte eine Lampe aus der Decke, auf der drei Farben wie bei einer Ampel übereinander angeordnet hatte: rot, gelb und grün. Bei grün lief die Produktionsanlage (,the machine") reibungslos, bei gelb war sie verlangsamt, bei rot stand sie. Sehr häufig kam es zu Stillständen der Maschine. Dann wurde ein Fehler gesucht, möglichst schnell repariert und es ging weiter. Die Reparaturen waren aber oft nicht gut, wie der zweite Werkleiter Robin, der Vorgesetzte der „Engineers“ bemerkte. In der Sitzung der „Engineers“, in Deutschland würde man sagen der Schlosser und Elektriker, die für die Reparaturen zuständig waren, offenbarte sich dann auch noch ein ziemlicher Konflikt. Das Klima zwischen ihnen war sehr schlecht. „Wenn der hier bleibt, werde ich gehen“ sagte ein Kollege auf einen anderen bezogen. Ich sprach mit Robin, dass da eine Konfliktklärung/Mediation nötig sei. Wir sprachen beide mit den beiden Hauptkontrahenten. Dabei passierte nichts Spektakuläres. Robin formulierte klar die Erwartungen als Führungskraft. Ich erzählte etwas über Professionalität und Mannschaftspiel im Fußball und dass man sich für gute Pässe zueinander nicht unbedingt lieben müsse. Die Kontrahenten unterschrieben nichts, schienen aber etwas nachdenklicher als vorher. So nahm ich an Sitzungen mit den Monteuren teil, die untereinander zum Teil sehr zerstritten waren (Beziehungsdynamik), und versuchte zur Mediation beizutragen.

\subsection{Sprache - „Housekeeping“}

Alle sahen, dass der Betrieb relativ unordentlich war. Dauernd fiel das Wort „Housekeeping“. Irgendwie störte mich der Begriff. Ich fragte dann in einer Schichtbesprechung die Arbeiter ,What is housekeeping for you?" Einer antwortete „Housekeeping is done by my lady at home“. Diese Konnotation des Begriffes erschien mir kontraproduktiv in einem so maskulinen Betrieb (118 Männer, zwei Frauen). Daher bat ich sie zu überlegen, um was es eigentlich ging. Herauskam, dass es eigentlich um drei klare produktionsrelevante Punkte ging.

1. Maintainance of the machine (ungestörter Lauf der Maschine)

2. Health and safety (Gesundheit und Sicherheit)

3. Less customer complaints (Weniger Kundenbeschwerden und Rückgaben)

Damit war „Housekeeping“ sinnvoll einem Reframing, einer Bedeutungsrevision, unterzogen. Reframíng stammt im strengen Sinne aus dem Neuro-Linguistischen Programmieren (Bandler und Grinder 2010), lässt sich aber für die Erweiterung des Bezugsrahmens (,Frame of reference“) gut nützen. Dies ist ein Beispiel für die Ergänzung der TA-Interventionen durch naheliegende aus anderen Ansätzen.

\subsection{Visualisierung II}

Die oben beschriebene Geschichte mit den Bildern brachte mich noch auf eine andere Idee, nämlich die zähen 14.00Uhr-Schichtbesprechungen aufzulockern, in denen bisher die Arbeiter in den Stühlen ,lagen“ und ein wenig eloquen- 
ter Schichtführer seiner Mannschaft etwas zu erzählen versuchte. Ich schlug ein Quiz mit Bildern von Situationen im Betrieb vor, die sie selber zusammentrugen, gemischt mit den polnischen und tschechischen Bildern, um nach obigen drei Kriterien die Situationen einzuordnen. Man stand dann um den Tisch herum, auf dem die Fotos lagen und diskutierte engagiert. Dies brachte Leben in die Schichtbesprechungen und trainierte den Blick für das Gewünschte. Natürlich kann man eine solche Vorgehensweise nicht ständig wiederholen. Sie wird auch irgendwann wieder langweilig. Aber sie freuten sich über etwas Neues und waren sehr engagiert.

\subsection{Abschied, dazwischen und der zweite Teil}

Von obiger Art Interventionen gab es eine Menge in den drei Tagen. Ich verließ dann nach drei Tagen die Fabrik, ,bis in drei Monaten'.

Kurz vor der zweiten Shadow-Woche rief ich vorsichtig beim deutschen Produktionsleiter an, ob sich schon irgendetwas verändert habe. Der deutsche Produktionsleiter sagte ,Durchaus, die Produktionszahlen sind durch die Decke gegangen. ' Der englische Werkleiter ergänzte ,You healed the machine". Ich freute mich, lies die ,esoterische“ Formulierung unkommentiert, war aber in mir unsicher, welche Intervention(en) - wenn überhaupt - genau dafür verantwortlich war.

Entsprechend versuchte ich nach meiner Rückkehr dorthin Anfang der zweiten Shadowing-Woche mit den Leuten herauszuarbeiten, was geholfen hatte, um dies zu ankern. Denn vom Standpunkt der Bewusstheit und Beeinflussbarkeit ist es sinnvoll zu wissen, wodurch positive Veränderungen erzielt werden.

Um es vorweg zu nehmen, so richtig fündig wurde ich nicht. Gut, sie freuten sich mich wiederzusehen. Meine Beziehungsqualität und damit vielleicht auch -wirkung schien vielleicht nicht so schlecht zu sein. Die positiven Bilder auf den Stellwänden waren da. Es sah etwas aufgeräumter aus. Das hatte aber vielleicht auch damit zu tun, dass vor ein paar Tagen der Auditor der Versicherung durch die Fabrik gegangen war und man deshalb aufgeräumt hatte.

Aber es stellte sich heraus: Die Mediationen zu Konflikten und Meetings mit den Elektrikern und Schlosser zur nachhaltigeren Reparatur von Schäden könnte eine mitentscheidende Intervention gewesen. Vor allem waren die Reparaturen deutlich besser geworden, insbesondere die am Wochenende, bei denen vorher geschludert worden war. Zudem waren die Verantwortungen klarer den verschiedenen Ebenen zugeordnet. Einer der Monteure war sehr viel besser drauf, weil sich bei ihm ein privates Problem gelöst hatte. So spielt sich vom Coachinginterventionsstandpunkt auch Glück eine wichtige Rolle.

\subsection{Was war nun Transaktionsanalyse an diesem Fall?}

Hier sind einige Punkte zu nennen:

1. Arbeit auf der Basis von Transaktionen (konkrete Interaktionen aufgreifen)

2. Radikaler Respekt den Leuten gegenüber (,What are you proud of?")

3. Betrachtung systemischer Verbindungen (Wie wirkt der Vorbildcharakter?)

4. Identifizierung von Mustern

5. Verwendung von Bildern, Visualisierung, Listen

6. Experimentelles Vorgehen (vieles probieren und in die Wege leiten)

7. Konfrontation von Abwertung, psychologischen Spielen und Symbiosen (Würdigung der Rollen)

8. Konfrontation von Bezugsrahmen (z. B. der Bezugsrahmen über Delegation und Vorbild)

9. Unterstützung der Bedürfnisse nach Anerkennung, Struktur und Stimulation

10. Unterstützung von Erwachsenen-Ich-Erwachsenen-IchBeziehungen

Dennoch gibt es für solche komplexen Situationen keine einfachen linearen Erklärungen für Veränderungen. Aber das unmittelbare Reagieren und Einfluss nehmen auf Interaktionen, das konstruktive Konfrontieren von Abwertungen (der Rollen, der Konflikte, ...) hatte offensichtlich Einfluss gehabt.

\section{Ausblick}

Die Welt verändert sich weiter. Dies betrifft materielle und geistige gesellschaftliche Entwicklungen. Für die TA hat dies mehrere Konsequenzen: Es gilt weiter im Sinne der Musterkonstruktion neue Konzepte zu entwickeln, die traditionellen Konzepte zu verfeinern, beispielsweise in den neuen Antreibern. Die guten, alten Antreiber, wie sie Taibi Kahler aus Little Rock, Arkansas in den 1970er Jahren beschrieben hatte, waren in seiner Zeit angemessen. Mittlerweile hat sich Einiges verändert „Sei auffallend!“ könnte einer der neuen gesellschaftlichen Maximen sein.

Für die Arbeit mit Systemdynamiken, die Kombination von systemischer Perspektive mit TA, liegen mittlerweile eine Menge Praxiserfahrungen in Unternehmen, öffentlichen Organisationen bis hin zu Kirchengemeinden vor. Ein frei verfügbarer Fragebogen (Scoring Instrument for the Systemic Organisational Analysis) lässt sich für die Klienten- wie auch für die Beraterperspektive nutzen (Mohr 2010). 
Interessenkonflikt G. Mohr gibt an, dass kein Interessenkonflikt besteht. G. Mohr hat keine finanziellen oder persönlichen Beziehungen zu Dritten, deren Interessen vom Beitragsinhalt positiv oder negativ betroffen sein könnten.

Open Access Dieser Artikel wird unter der Creative Commons Namensnennung 4.0 International Lizenz veröffentlicht, welche die Nutzung, Vervielfältigung, Bearbeitung, Verbreitung und Wiedergabe in jeglichem Medium und Format erlaubt, sofern Sie den/die ursprünglichen Autor(en) und die Quelle ordnungsgemäß nennen, einen Link zur Creative Commons Lizenz beifügen und angeben, ob Änderungen vorgenommen wurden.

Die in diesem Artikel enthaltenen Bilder und sonstiges Drittmaterial unterliegen ebenfalls der genannten Creative Commons Lizenz, sofern sich aus der Abbildungslegende nichts anderes ergibt. Sofern das betreffende Material nicht unter der genannten Creative Commons Lizenz steht und die betreffende Handlung nicht nach gesetzlichen Vorschriften erlaubt ist, ist für die oben aufgeführten Weiterverwendungen des Materials die Einwilligung des jeweiligen Rechteinhabers einzuholen.

Weitere Details zur Lizenz entnehmen Sie bitte der Lizenzinformation auf http://creativecommons.org/licenses/by/4.0/deed.de.

\section{Literatur}

Apphat (Asociación de Profesionales de Psicología Humanista y Análisis Transaccional) (2021). Effectivity of transactional analysis. Controlled studies, RCT design studies and meta-analysis

Balling, R. (1989). Manipulation, Motipulation, Motivation. Oder: „Wie kriege ich Deine Arbeitskraft?“. Zeitschrift für Transaktionsanalyse, ZTA, 6(2-3), 109-119.

Balling, R. (2005). Diagnose von Organisationskulturen. Zeitschrift für Transaktionsanalyse, ZTA, 22(4), 234-253.

Bandler, R., \& Grinder, J. (2010). Reframing (10. Aufl.). Paderborn: Junfermann.

Berne, E. (1979). Struktur und Dynamik von Organisationen und Gruppen. München: Kindler. (1963/1979)

Berne, E. (2005). Grundlagen der Gruppenbehandlung: Grundgedanken und Interventionstechniken. Paderborn: Junfermann. $(1961 / 2005)$

Brunner, K., \& Sell, M. (Hrsg.). (2021). Transaktionsanalytische Supervision in Theorie und Praxis. Paderborn: Junfermann.

Buber, M. (1983). Ich und Du. Heidelberg: Lambert Schneider.

Bundesärztekammer - Bekanntmachungen, Wissenschaftlicher Beirat Psychotherapie nach $\S 11$ PsychThG, Gutachten zur wissenschaftlichen Anerkennung der HumanistischePsychotherapie, Deutsches Ärzteblatt 9. März 2018

Cornell, B., de Graaf, A., Newton, T., \& Thunissen, M. (2016). Into TA-A comprehensive textbook on transactional analysis. London: Karnac.

Dehner, U., \& Dehner, R. (2013). Transaktionsanalyse im Coaching. Bonn: mangerseminare.

Deutscher Bundesverband Coaching e.V. (Hrsg.). (2012). Coaching als Profession, Kompendium mit den Professionsstandards des $D B V C$ (4. Aufl.). Osnabrück: DBVC-Geschäftsstelle.

Eidenschink, K. (2016). Veränderung verstehen. https://metatheorieder-veraenderung.info/wp-content/uploads/2016/02/Veraenderungverstehen.pdf. Zugegriffen: 5. Mai 2021.

English, F. (1972a). The substitution factor. Rackets and real feelings. Part I. Transactional Analysis Journal, 1(4), 27-33.

English, F. (1972b). The substitution factor. Rackets and real feelings. Part II. Transactional Analysis Journal, 2(1), 23-25.

English, F. (1975). The three-cornered contract. Transactional Analysis Journal, 5(4), 383-384.
Erskine, R. G., Moursund, J.P., \& Trautmann, R. L. (1999). Beyond empathy: A therapy of contact-in-relationship. Philadelphia: Brunner-Routledge.

Federn, P. (1953). Ego psychology and psychosis. New York: Basic Books. (dt.: Ichpsychologie und die Psychosen, Frankfurt: Suhrkamp 1978)

Fox, E. (1975). Berne's organizational theory. Transactional Analysis Journal, 5(4), 345-353.

Fritzsche, K., \& Hartmann, W. (2010). Einführung in die Ego-StateTherapie. Heidelberg: Auer.

Goulding, M. M., \& Goulding, R. L. (2005). Neuentscheidung: Ein Modell der Psychotherapie (7. Aufl.). Stuttgart: Klett-Cotta.

Hagehülsmann, U., \& Hagehüsmann, H. (1998). Der Mensch im Spannungsfeld seiner Organisation. Paderborn: Junfermann.

Hallstein, G. (2021). STeP: Supervision und Training in ethischer Professionalität. In K. Brunner \& M. Sell (Hrsg.), Transaktionsanalytische Supervision in Theorie und Praxis (S. 21-41). Paderborn: Junfermann.

Hauser, H.-G. (1991). Unternehmenskultur aus transaktionsanalytischer Sicht. Zeitschrift für Transaktionsanalyse, ZTA, 8(4), 179-192.

Hay, J. (1995). Transactional analysis for trainers. Minneapolis: Sherwood.

Hay, J. (2007). Reflective practice and supervision for coaches. New York: McGraw Hill-Open University Press.

Höher, F. (2020). Menschliche Resilienz in Unternehmen - Dialog als Ressource. Opladen: Barbara Budrich.

Honneth, A. (1992). Kampf um Anerkennung. Frankfurt a. M.: Suhrkamp.

Horn, E. K., Verheul, R., Thunnissen, M., Delimon, J., \& Soons, M. (2015). Effectiveness of short-term inpatient psychotherapy based on transactional analysis with patients with personality disorders: a matched control study using propensity score personality disorders. Journal of Personality Disorder, 29(5), 663-683.

Kessel, B., Raeck, H., \& Verres, D. (2020). Ressourcenorientierte Transaktionsanalyse. Göttingen: Vandenhoek \& Ruprecht.

Korpiun, M. (2020). Relational organizational development. Transactional Analysis Journal, 50(3), 207-220.

Lippman, E. (Hrsg.). (2013). Coaching - Angewandte Psychologie für die Beratungspraxis. Berlin: Springer.

Looss, W. (1996). Coaching für Manager. Problembewältigung unter vier Augen. München: Verlag moderne Industrie.

Mohr, G. (2000). Lebendige Unternehmen führen. Frankfurt a.M.: FAZ-Buchverlag.

Mohr, G. (2005). Transactional analysis identity. The Script, 35(4), $1-3$.

Mohr, G. (2006). Systemische Organisationsanalyse. Bergisch Gladbach: EHP.

Mohr, G. (2008). Coaching und Selbstcoaching mit Transaktionsanalyse. Bergisch Gladbach: EHP.

Mohr, G. (2010). Workbook Coaching und Organisationsentwicklung. Bergisch Gladbach: EHP.

Mohr, G. (2012). Pattern theory as a meta concept for change. Transactional Analysis Journal, 42(2), 134-147.

Mohr, G. (2013). Achtsamkeitscoaching. Bergisch Gladbach: EHP.

Mohr, G. (2014). Systemic transactional analysis coaching: a study of effective conditions, consequences and effects on organisational culture. International Journal of Transactional Analysis Research \& Practice, 5(2), 3-16.

Mohr, G. (2015). Systemische Wirtschaftsanalyse. Bergisch Gladbach: EHP.

Mohr, G. (2017). Resilienzcoaching für Menschen und Systeme. Bergisch Gladbach: EHP.

Mohr, G. (2019). An application of Berne's organizational boundary theory and a quota for women on company boards. Transactional Analysis Journal, 49(1), 43-52.

Mohr, G. (2020). Einführung in die systemische Transaktionsanalyse von Individuum und Organisation. Heidelberg: Carl-Auer. 
Möller, H., \& Kotte, S. (2013). Diagnostik im Coaching. Berlin, Heidelberg: Springer.

Mountain, A., \& Davidson, C. (2015). Working together-Organizational transactional analysis and business performance. Farnham: GB (Gower).

Peichl, J. (2019). Einführung in die hypnosystemische Teiletherapie. Heidelberg: Carl-Auer.

Pratt, K. (2021). Transactional analysis coaching. New York: Routledge.

Rautenberg, M. (2020). Zen in der Kunst des Coachings. Heidelberg: Carl-Auer.

Rosa, H. (2016). Resonanz. Eine Soziologie der Weltbeziehung. Berlin: Suhrkamp.

Roth, G., \& Ryba, A. (2016). Coaching, Beratung und Gehirn - Neurobiologische Grundlagen wirksamer Veränderungskonzepte. Stuttgart: Klett-Cotta.

Ryba, A. (2018). Die Rolle unbewusster und unbewusst-intuitiver Prozesse im Coaching. Göttingen: Vandenhork \& Ruprecht.

Schiff, J.L., et al. (1975). Cathexis reader. Transactional Analysis Treatment of Psychosis. London: Harper \& Row.

Schmid, B. (1986). Systemische Transaktionsanalyse. Wiesloch: Eigenverlag B. Schmid.

Schmid, B. (1989). Programmatische Überlegungen anlässlich der Entgegennahme des 1. EATA-Wissenschaftspreises für Autoren (Blackpool 1988). Zeitschrift für Transaktionsanalyse, 6(4), 141-163.

Schmid, B. (1994). Wo ist der Wind, wenn er nicht weht .... Paderborn: Junfermann.

Schmid, B. (2003). Systemische Professionalität und Transaktionsanalyse. Bergisch Gladbach: EHP.

Schneider, J. (2000). Supervidieren \& beraten lernen. Paderborn: Junfermann.

Schreyögg, A. (2012). Die Wissensstruktur von Coaching, in: DBVC, Coaching als Profession, Kompendium mit den Professionsstandards des DBVC (4. Aufl.). (S. 106-113). Osnabrück: DBVC.
Schulze, H., \& Sejkora, K. (2015). Positive Führung - Resilienz statt Burnout. Freiburg: Haufe.

Sedgwick, J. M. (2021). Contextual transactional analysis. New York: Routledge.

Sejkora, K., \& Schulze, H. (2017). Vom Lebensplan zum Beziehungsraum: Wie Sie mit Hilfe der Transaktionsanalyse begrenzende Muster überwinden. Munderfing: Fischer \& Gann.

Sills, C., \& Hargarden, H. (2003). Ego states, key concepts in transactional analysis. London: Worth Publishing.

Temple, S. (2002). Functional Fluency. Zeitschrift für Transaktionsanalyse, 4, 251-269.

Tudor, K., \& Summers, G. (2014). Co-creative transactional analysis. London: Karnac Books.

Van Rijn, B., \& Wild, C. (2016). Comparison of transactional analysis group and individual psychotherapy in treatment of depression and anxiety. Transactional Analysis Journal, 46(1), 63-74.

Vogelauer, W. (1991). Unternehmenskultur aus transaktionsanalytischer Sicht: „Organisationspersönlichkeit und OrganisationsSkript“. Zeitschrift für Transaktionsanalyse, ZTA, 8(4), 193-206.

Vos, J., \& van Rijn, B. (2021). The evidence-based conceptual model of transactional analysis: a focused review of the research literature. Transactional Analysis Journal. https://doi.org/10.1080/ 03621537.2021.1904364.

Žvelc, G., Černetič, M., \& Košak, M. (2011). Mindfulness-based transactional analysis. Transactional Analysis Journal, 41(3), 241-254.

Publisher's Note Springer Nature remains neutral with regard to jurisdictional claims in published maps and institutional affiliations. 